\title{
Ischemic stroke in a patient with interatrial block: Is it only a coincidence or a clear relationship?
}

\author{
Athanassios Antonopoulos ${ }^{1}$, Laila Fiorani ${ }^{2}$

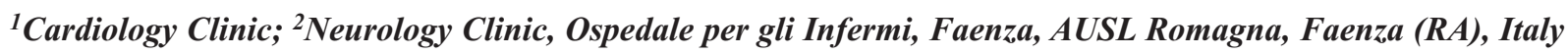

\begin{abstract}
Atrial fibrillation (AF) can be detected in nearly $25 \%$ of all patients with stroke by sequentially combining different electrocardiographic methods. Prediction of early cardio-embolic stroke remain a permanent challenge in everyday practice. The early identification of an increased risk for atrial fibrillation episodes (which are frequently asymptomatic) is essential for the
\end{abstract}

Correspondence: Athanassios Antonopoulos, UO Cardiologia,

Ospedale per gli Infermi, Viale Stradone 9, 48018 Faenza (RA), Italy. Tel. +39.0546.601238 - Fax: +39.0546.601110.

E-mail: athanassios.antonopoulos@auslromagna.it

Key words: Interatrial block; stroke; cerebral ischemia; atrial fibrillation; oral anticoagulation.

Authors' contributions: AA, began the project, wrote, review the manuscript, and the figures looked for and carefully examined the references and approved the final manuscript; LF, collected patient data, evaluated her clinical course and treatment. All authors have read and approved the final version of the manuscript and agree to be accountable for all aspects of the work.

Conflict of interest: The authors report no conflicts of interest or funding support.

Ethics approval and consent to participate: No ethical committee approval was requires for this case report by the Departments, because this article does not contain any studies with human participants or animals.

Informed consent: consent of EKG exam was obtained from the patient included in this study.

Consent for publication: not required as each EKG and event recorder image are anonymized and therefore the confidentiality or personal data is guaranteed.

Received for publication: 22 November 2020 .

Accepted for publication: 5 January 2021.

${ }^{\circ}$ Copyright: the Author(s), 2021

Licensee PAGEPress, Italy

Monaldi Archives for Chest Disease 2021; 91:1692

doi: 10.4081/monaldi.2021.1692

This article is distributed under the terms of the Creative Commons Attribution Noncommercial License (by-nc 4.0) which permits any noncommercial use, distribution, and reproduction in any medium, provided the original author(s) and source are credited. prevention of cardioembolic events. One of the noninvasive modalities of atrial fibrillation prediction is represented by the electrocardiographic P-wave analysis. This includes study and diagnosis of interatrial conduction block. Our short case report presents a case with ischemic cortico-sottocortical stroke involving capsulo and caudo regions in a woman patient with interatrial block as realized by electrocardiographic $\mathrm{P}$ analysis.

\section{Introduction}

Description of the interatrial conduction blockade in 1979, classifying it into interatrial and intra-auricular blocks, suggested separating interatrial blocks (IAB) into two categories: a) partial, where there would be a delay in the conduction of the Bachmann's region but most of the right-to-left conduction still occurs at an auricular ceiling level; and b) advanced where there is a total block of Bachmann's region and the conduction towards the left atrium occurs from the lower part of the right atrium with a caudo-cranial retrograde direction mainly through the coronary sinus, and to a lesser extent through the fossa ovallis [1]. Moreover, IAB seems to be a key factor of atrial fibrillation (AF). Diagnosis of IAB are easy to perform using the surface ECG. It is partial when the P wave duration is $\geq 120 \mathrm{~ms}$, and advanced if the $P$ wave presents a biphasic pattern in II, III and aVF. IAB are very frequent in the elderly and, particularly in the case of the advanced type, they are associated with $\mathrm{AF}$, and $\mathrm{AF}$ recurrences [2]. This association has been recently named Bayés syndrome [3]. Moreover, IAB increases risk of stroke [4], and seems to be associated with dementia [5]. Although the reasons for this association are still not clear, atrial remodeling due to delayed and abnormal left atrium activation, especially in case of advanced IAB, is probably the key factor in the chain of events that lead to atrial fibrosis.

We describe a case of a patient in whom detection of aIAB motivated extended cardiac monitoring resulting in the diagnosis of paroxysmal AF and a subsequent decision on full oral anticoagulation strategy.

\section{Case Report}

In September 2020 an 80-year-old woman patient with hypertension under treatment and chronic coronary syndrome revascularized in 2007 by coronary angioplasty in right coronary and left anterior descending coronary arteries with 2 stents placement, arrived in emergency room after an episode of syncope and fall. No palpitations, angina or dyspnea had been observed in the last 3 months. In June 2020 the patient performed an 
echocardiography which had shown an hypokinesia in the inferior wall with normal biventricular global systolic function. Initial degeneration of mitral and aortic cardiac valves with gradients max/medium of $16 / 10 \mathrm{mmHg}$ in aortic valve. Both right and left atrial were normal. During that period patient started with the following therapy: Metoprolol $25 \mathrm{mg}$ twice daily, atorvastatin 40 $\mathrm{mg}$ once daily, foline $5 \mathrm{mg}$ once daily, ramipril $5 \mathrm{mg}$ once daily, hydroclorothizide $25 \mathrm{mg}$ once daily.

The days before her admission, patient was functionally independent with a good performance status. Her physical exam had shown a systolic heart murmur $2 / 6$ with carotid irradiation. EKG was performed with sinus bradycardia (55 bpm), P duration $124 \mathrm{ms,}$ PQ $182 \mathrm{~ms}$, QTc 392ms. Moreover, cardiac telemetry did not show any evidence of arrhythmia. A Carotid Doppler ultrasound was negative for significant stenosis, but only bilateral atherosclerotic plaques were present. A transthoracic echocardiogram was negative for thrombus or severe atrial dilation; A Neurology evaluation performed revealed a left hemiparesis with partial disorientation in the space and time and hemiplegia mainly of the left superior art. CT of the head did not show any acute changes to explain the patient's symptoms. Then the patient was admitted in the Neurology clinic for clinical and therapeutic FU.

Initial laboratory results were $\mathrm{Hb} 12.3 \mathrm{~g} / \mathrm{dl}$, PLT 211000 , creatinine $0.75 \mathrm{mg} / \mathrm{dl}$, total cholesterol $159 \mathrm{mg} / \mathrm{dl}$, LDL 90mg/dl, triglycerides $129 \mathrm{mg} / \mathrm{dl}$, TSH $2.960 \mathrm{mU} / \mathrm{L}$, uric acid $3.9 \mathrm{mg} / \mathrm{dl}$, total bilirubin $0.63 \mathrm{mg} / \mathrm{dl}$, ALT 10U/L, Gamma GT $16 \mathrm{U} / \mathrm{L}$, within normal limits. In the following day MRI with gadolinium of the brain revealed multifocal mildly increased diffusion signals, involving capsulo-lenticular and thalamic right regions. Moreover, another focus in the right temporal in sottocortical region and in right nucleus caudatum level, confirming multifocal ischemic stroke. Her CHA2DS2VASc score was 5.

A transesophageal cardiac ultrasound was performed without a presence of thromboembolic formations. During the patient hospitalization a Holter EKG monitoring was performed without signs of atrial fibrillation or flutter. Another bedside EKG was performed without any significant changes (Figure 1). A careful observation of the P wave morphology, duration and voltage revealed an advanced interatrial block alteration (duration $124 \mathrm{~ms}$, and the polarity was bimodal in II lead and biphasic in the III, aVF inferior leads and $\mathrm{P}$ wave axis normal, i.e. +23 degrees). The prediction of atrial fibrillation we obtained by applying the simple electrocardiographic MVP ECG risk score [6]. which show a score equal do 5 (high probability risk of atrial fibrillation). The above score takes into consideration the morphology, voltage and duration of Pwave for the prediction of AF. So, due to high prediction AF score, before initializing anticoagulant therapy, it was considered important to demonstrate an episode of AF. For this purpose we repeated a 7day EKG registration by an external event EKG recorder (Figure 2). After this prolong time EKG monitoring, we discover multiple, but short periods of atrial fibrillation. So, we decide to initiate anticoagulant therapy. Furthermore, during the hospitalization staying the patient underwent physical and occupational therapy and she achieved improvement in her symptoms.

\section{Discussion}

The embolism of cardiac origin is a common cause of ischemic stroke. AF can be discovered in nearly $25 \%$ of all patients with stroke by sequentially combining different electrocardiographic methods [1]. Moreover, advanced IAB (aIAB) have been described to predict occurrence of new onset and recurrent AF [2]. The prevalence of aIAB and partial IAB is $1 \%$ and $9.7 \%$ respectively in the general population and they are both associated with increased risk of AF [7]. Its prevalence increases with age, reaching a prevalence of $26 \%$ (aIAB) and 20,1\% (partial IAB) in subjects aged $>100$ years [5]

Aging is associated with a progressive increase in the degree of atrial fibrosis and modification in the cardiac conduction system, which led to IAB. AF, atrial premature beats and runs of atrial arrhythmias are also associated with age and with IAB [8]. Sometimes also, from the 12-leads surface EKG an abnormal frontal $\mathrm{P}$ wave axis change may be associated with long-term risk prediction of AF. In the ARCS study the risk of AF increased as P axis became $<14^{\circ}$ and $>55^{\circ}$. In this case, P-wave axis was normal. In addition, shifts in the $\mathrm{P}$ wave axis have been linked with atrial enlargement, an established AF factor [9] on echocardiography and MRI [10] but in the present patient atrial dimensions were normal. Additionally, Cotter and coworkers were observed a rapid increase of IAB prevalence from middle age to senescence. The last probably due to the degree of atrial fibrosis and the gradual modification of the specialized cardiac conduction system. In contrast, in young adults the prevalence of IAB is low, except in patients with cryptogenic stroke and patent foramen ovale that can be of $46 \%$ [11]. Moreover, in cardiac magnetic resonance and late gadolinium enhancement of the upper part of the septum involving Bachmann's bundle has been reported, describing the association between $\mathrm{IAB}$ and atrial fibrosis [12]. As a result of the above condition an endothelium dysfunction may be present, with a hypercoagulable state comparable to that present in AF [13] and a stagnant and sluggish left atrium favoring the appearance of stasis -induced thrombosis, especially in the left appendage, even in absence of supraventricular arrhythmias. In the present case, cardiac telemetry did not show any evidence of arrhythmia, but a high CHA2DS2VASc-score was observed (equal to 5) while no other previous arrhythmic events were reported.

Accordingly, some studies also suggest that patients with high CHA2DS2VASc-score and IAB have a high stroke risk irrespective of the presence of AF [14,15]. These data support the notion that $\mathrm{AF}$ is a risk factor for ischemic stroke, but not necessarily the direct cause of it.

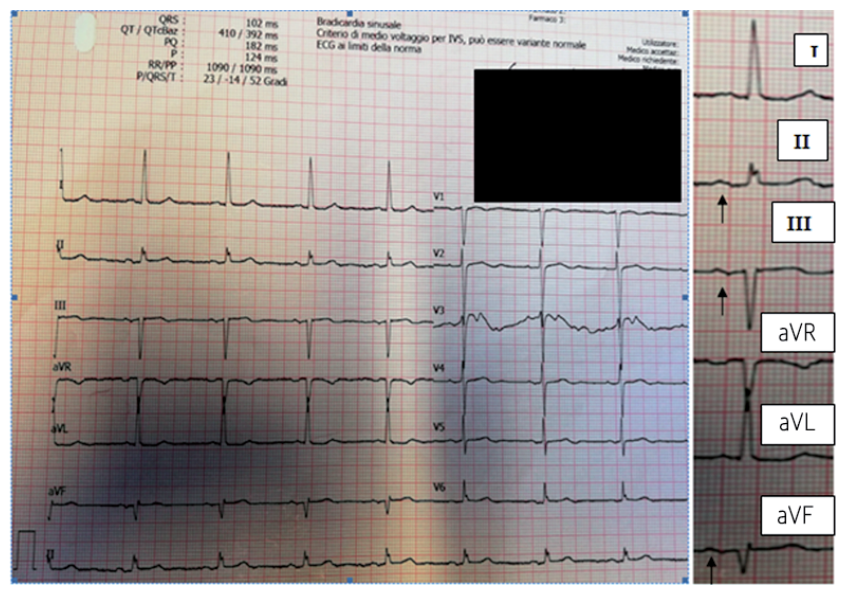

Figure 1. ECG: 12-lead ECG $(10 \mathrm{~mm} / \mathrm{mV}, 25 \mathrm{~mm} / \mathrm{s})$ at follow-up. ECG shows sinus rhythm. In the sinus beats, $P$ wave duration is increased (124 ms), $P$ waves have a biphasic morphology in leads III and aVF, and a bimodal morphology in lead II, consistent with advanced $I A B$ with a $P$ wave axis +23 degrees (normal). 


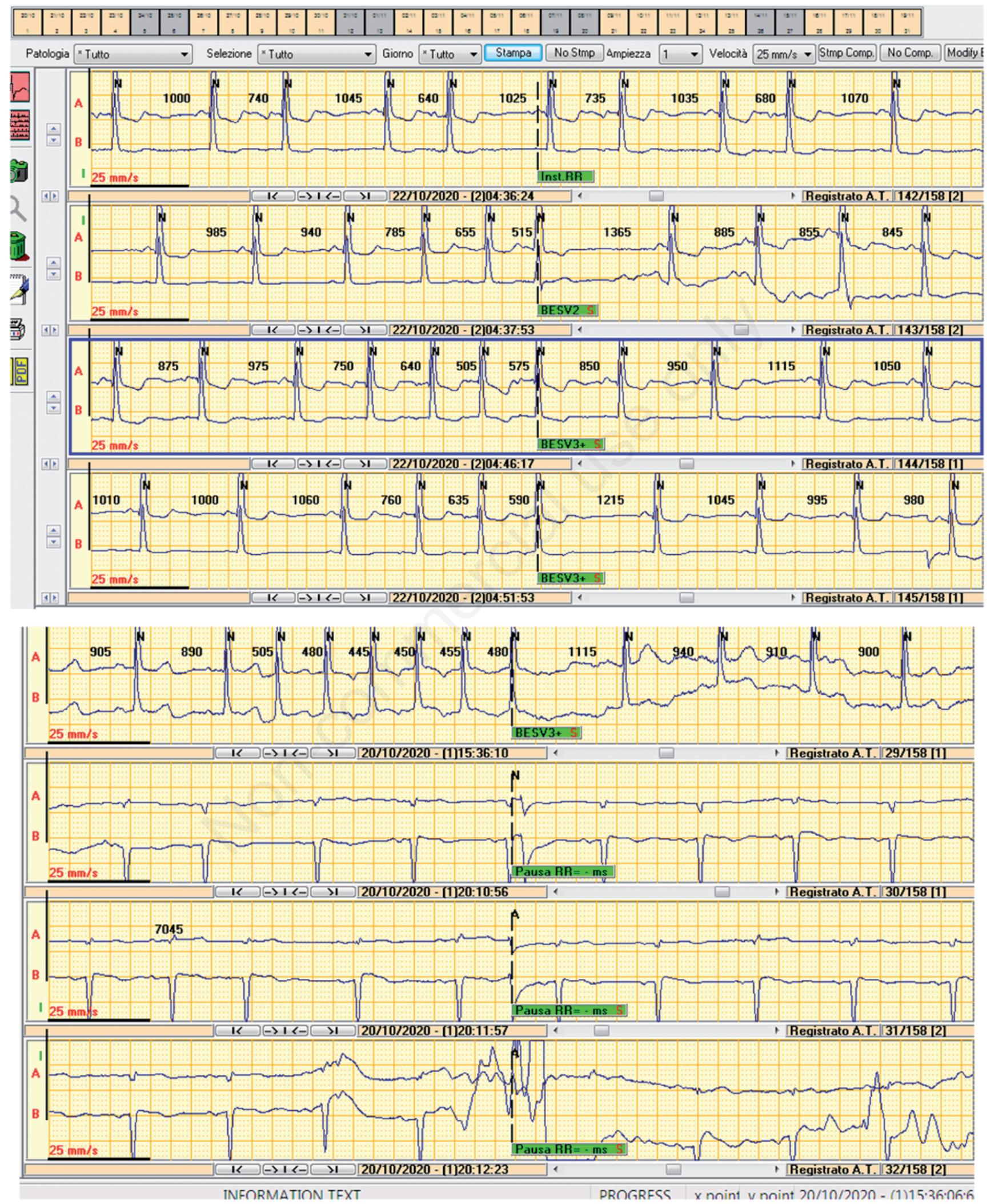

Figure 2. Some strips with episodes of paroxysmal atrial fibrillation were recorded in the ECG external event recorder monitoring. 
On the other hand, an association between advanced IAB and supraventricular arrhythmias and poor left atrium contractility has been reported. This is called Bayes' Syndrome [16]. This association may be explained by atrial fibrosis and left atrium enlargement. Also, this could be due to re-entry, as conduction disturbances increase refractory period dispersion. Finally, IAB is associated with premature atrial beats probably due to abnormal left atrium activation facilitating the initiation of re-entry and AF [17].

Despite the recent appreciation of IAB as a predictor of atrial fibrillation IAB is still underused in everyday clinical practice. On the other hand, the bedside ECG seems to be the simplest and most appropriate method that could aid clinicians to appreciate and recognize $\mathrm{IAB}$ and predict $\mathrm{AF}$. In this case, was used to investigate the potential explanation of an ischemic stroke beyond the presence of a clear AF and eventually to decide for anticoagulant therapy initiation. It is well known that patients with advanced IAB without previous episodes of documented AF also have an increased risk of stroke, particularly in the presence of high CHA2DS2VASc score, and/or structural heart disease. However, at this time, we do not have clinical trials that support the use of anticoagulants in the absence of documented AF. Therefore, it is important to carry out monitoring to look for AF episodes that can support anticoagulation in these patients $[18,19]$. In fact, in considered patient, after a longtime monitoring, we found short repetitive runs of AF that support anticoagulation therapy. In conclusion, advanced IAB seems to be a risk factor for ischemic stroke even in the absence of documented AF especially if coexist a high CHA2DS2VASc score. A high MVP ECG score may predict future AF events. For anticoagulant therapy decision it is essential to assess future studies which help to elucidate whether anticoagulation is needed in these patients.

\section{References}

1. Conde D, Seoane L, Gysel M, et al. Bayés' syndrome: the association between interatrial block and supraventricular arrhythmias. Expert Rev Cardiovasc Ther 2015; 13:541-50.

2. Bayés de Luna A, Cladellas M, Oter R, et al. Interatrial conduction block and retrograde activation of the Left Atrium and paroxysmal supraventricular tachyarrhythmia. Eur Heart J 1988;9:1112-8.

3. Conde D, Baranchuk A. Interatrial block as anatomicalelectrical substrate for supraventricular arrhythmias: Bayés syndrome. Arch Cardiol Mex 2014;84:32-40.

4. Spodick DH, Ariyarajah V. Interatrial block: the pandemic remains poorly perceived. Pacing Clin Electrophysiol 2009;32:667-72.

5. Martínez-Sellés M, Massó-van Roessel A, Álvarez-García J, et al. Interatrial block and atrial arrhythmias in centenarians:
Prevalence, associations, and clinical implications. Heart Rhythm 2016;13 645-51.

6. Bryce A, Milden J, Bachar H, et al. New electrocardiographic score for the prediction of atrial fibrillation: The MVP ECG risk score (morphology-voltage-P-wave duration). Ann Noninvasive Electrocardiol 2019;24:e12669.

7. Istolahti T, Eranti A, Huhtala H, et al. The prevalence and prognostic significance of interatrial block in the general population. Ann Med 2020;20:1-23.

8. Martínez-Sellés M. Prevalence and incidence of interatrial block in global population and in different clinical situations. J Geriatr Cardiol 2017;14:158-60.

9. Maheshwari A, Norby FL, Soliman EZ, et al. Refining prediction of atrial fibrillation risk in the general population with analysis of P-wave axis (from the Atherosclerosis Risk in Communities Study). Am J Cardiol 2017;120:1980-4.

10. German MD, Kabir MM, Dewland TA, et al. Atrial fibrillation predictors: Importance of the electrocardiogram. Ann Noninvasive Electrocardiol 2016;21:20-9.

11. Cotter PE, Martin PJ, Pugh PJ, et al. Increased incidence of interatrial block in younger adults with cryptogenic stroke and patent foramen ovale. Cerebrovasc Dis Extra 2011;1:36-43.

12. Benito EM, De Luna AB, Baranchuk A, et al. Extensive atrial fibrosis assessed by late gadolinium enhancement cardiovascular magnetic resonance associated with advanced interatrial block electrocardiogram pattern. Europace 2017;19:377.

13. Larsen BS, Kumarathurai P, Falkenberg J. Excessive atrial ectopy and short atrial runs increase the risk of stroke beyond incident atrial fibrillation. J Am Coll Cardiol 2015;66:232-41.

14. Tischer Ts, Schneider R, Lauschke J, et al. Prevalence of atrial fibrillation in patients with high CHADS2- and CHA2DS2VASc-scores: anticoagulate or monitor high-risk patients? Pacing Clin Electrophysiol 2014;37:1651-7.

15. Zuo ML, Liu S, Chan KH, et al. The CHADS2 and CHA 2DS 2-VASc scores predict new occurrence of atrial fibrillation and ischemic stroke. J Interv Card Electrophysiol 2013;37:47-54.

16. Bacharova L, Wagner GS. The time for naming the interatrial block Syndrome: Bayes Syndrome. J Electrocardiol 2015;48:133-4.

17. Kumarathurai P, Mouridsen MR, Mattsson N, et al. Atrial ectopy and $\mathrm{N}$-terminal pro-B-type natriuretic peptide as predictors of atrial fibrillation: a population-based cohort study. Europace 2017; 19:364-70.

18. Bayés de Luna A, Martínez-Sellés M, Bayés-Genís A, et al. Surface ECG interatrial block-guided treatment for stroke prevention: rationale for an attractive hypothesis. BMC Cardiovasc Disord 2017; 17:211.

19. Martínez-Sellés M, Fernández Lozano I, Baranchuk A, et al. Should we anticoagulate patients at high risk of atrial fibrillation? Rev Esp Cardiol 2016;69:374-6. 